\title{
Noise-Induced Breakdown of Stochastic Resonant Behavior of van der Pol Oscillators Coupled by Time-Varying Resistor
}

\author{
Yoko Uwate \\ Institute of Neuroinfomatics, \\ University / ETH Zurich, \\ Winterthurerstrasse 190, \\ CH-8057 Zurich, Switzerland, \\ Email: yu001@ini.phys.ethz.ch
}

\author{
Yoshifumi Nishio \\ Dept. of Electrical and Electronic Eng., \\ Tokushima University \\ 2-1 Minami-Josanjima, Tokushima, Japan \\ Email: nishio@ee.tokushima-u.ac.jp
}

\author{
Ruedi Stoop \\ Institute of Neuroinfomatics, \\ University / ETH Zurich, \\ Winterthurerstrasse 190, \\ CH-8057 Zurich, Switzerland, \\ Email: ruedi@ini.phys.ethz.ch
}

\begin{abstract}
We explore the behavior of two van der Pol oscillators coupled by a stochastically time-varying resistor. We observe switching between in-phase and anti-phase synchronization and analyze the statistics of the switching, with and without additional noise. We find stochastic resonant behavior of synchronization in both regimes. Correlating the two noise sources quickly destroys the stochastic resonance phenomenon in the anti-phase synchronization regime. In the in-phase regime, noise correlation first contributes to the synchronization before removing the resonance by taking over the synchronization by its own means.
\end{abstract}

\section{INTRODUCTION}

Coupled oscillatory circuits provide simple models for describing high-dimensional nonlinear phenomena occurring in our everyday world. Synchronization, in particular, is one of the most important features that can be described and explored with the help of oscillators, because, upon their coupling, strongly correlated rhythms among the oscillators emerge, called synchronized states. Synchronization phenomena have been extensively reported in physical [1]-[4], biological [5],[6] and electrical [7],[8] systems.

Synchronization in networks of chaotic cells with stochastically switched couplings ('blinking networks') have been first reported in the context of the coupling among smallworld networks [9], where the authors sketched some potential applications of this model for explorations in neuroscience. In a previous research, we applied the blinking coupling concept for the coupling among van der Pol oscillators. The oscillators were coupled by means of a time-varying resistor, implemented by periodically switching between a positive and a negative resistor [10], as shown in Fig. 1. Using computer simulations, we confirmed the coexistence [11] of the in-phase (see. Fig. 2) and the anti-phase (see. Fig. 3) synchronizations, which depend upon the provided initial conditions [12]. This coexistence phenomenon of synchronization is interesting, since in resistor-coupled systems of two oscillators, even if multiple synchronization states exist, normally only one synchronization state becomes stable. The observed phenomenon may be of technical importance as it may open avenues for novel strategies of parallel information processing.

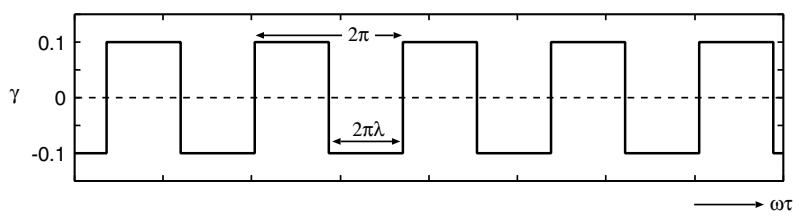

Fig. 1. Characteristics of the TVR.

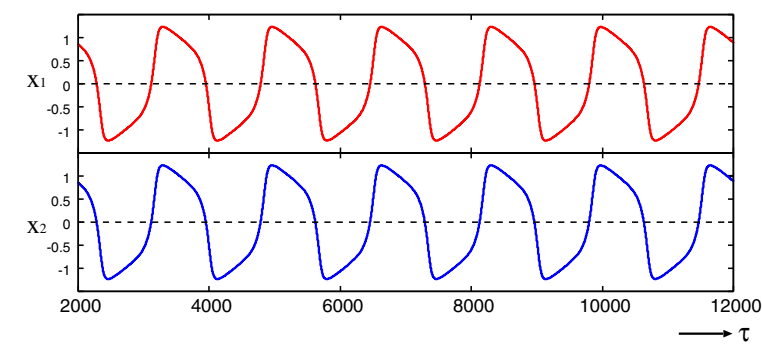

Fig. 2. In-phase synchronization.

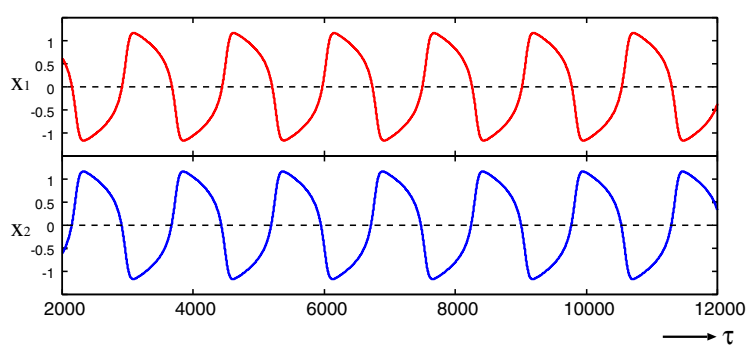

Fig. 3. Anti-phase synchronization.

In this contribution, we analyze the behavior obtained from a new type of time-varying resistor, where the state of the time-varying resistor is determined from an event probability, a setting that we propose to call "Stochastically Time-Varying Resistor (STVR) coupling". In this setting, switching between 
the in- phase and the anti-phase synchronized solutions is observed, similar to findings related with other systems [13]. In order to obtain a more detailed picture of the phenomenon, the sojourn time into the in-phase vs. the anti-phase synchronized regime is investigated, as a function of the parameters of STVR. In order to mimic the real world behavior, we add noise to the capacitors involved in each of the circuits. We find that the in-phase and anti-phase regime sojourn times depend inversely upon the noise strength, when identical noise $\xi_{1}=\xi_{2}$ are used for the two circuits. This discrepancy is gradually destroyed if the noise in the the two oscillators is made ever more independent.

\section{Circuit Model}

We study a circuit of two (up to later added noise) identical van der Pol oscillators, coupled by a stochastically timevarying resistor (STVR), see Fig. 4. The characteristics of the STVR is shown in Fig. 5. In the time interval $\left[k \pi / \omega_{t}\right.$, $\left.(k+1) \pi / \omega_{t}\right], R(t)$ is piecewise constant, taking one of the values $\{r,-r\}$. The event probabilities $p_{+}$and $p_{-}$for tasking the values $r$ or $-r$, respectively, satisfy the equation

$$
p_{+}+p_{-}=1.0 \text {. }
$$

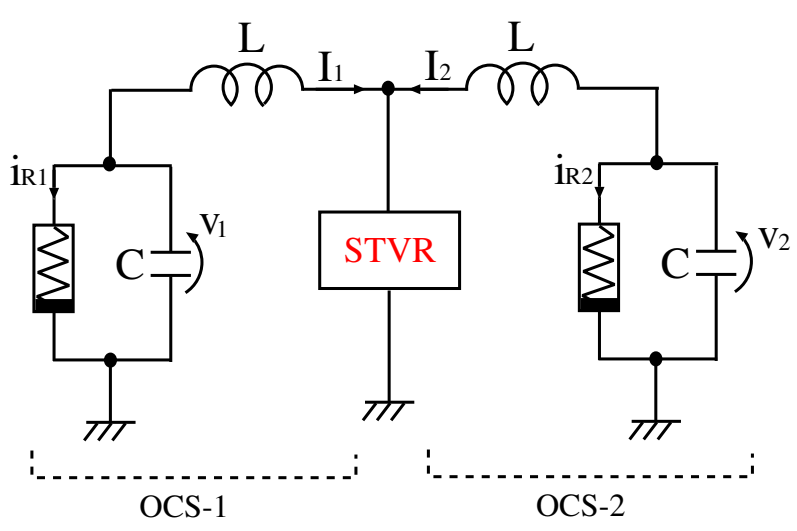

Fig. 4. Circuit Model (STVR is a Stochastically Time-Varying Resistor).

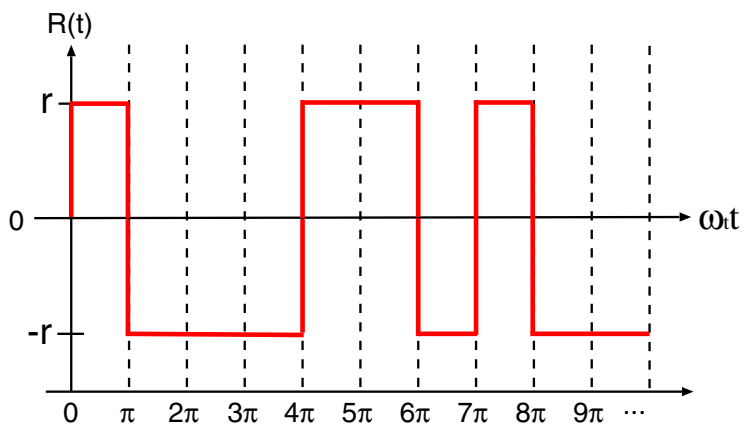

Fig. 5. Characteristics of STVR.

The van der Pol oscillator consists of an active element (nonlinear resistor) characterized by a simple symmetric cubic nonlinearity of the form

$$
i_{R k}=-g_{1} v_{k}+g_{3} v_{k}^{3}, \quad g_{1}, g_{3}>0, \quad k=1,2 .
$$

By a change of variables and parameters

$$
\begin{gathered}
v_{k}=\sqrt{\frac{g_{1}}{g_{3}}} x_{k}, \quad i_{R k}=\sqrt{\frac{g_{1}}{3 g_{3}}} \sqrt{\frac{C}{L}} y_{k}, \quad t=\sqrt{L C} \tau, \\
\varepsilon=g_{1} \sqrt{\frac{L}{C}}, \quad \gamma=r \sqrt{\frac{C}{L}}, \quad \omega=\frac{1}{\sqrt{L C}} \omega_{t},
\end{gathered}
$$

The normalized circuit equations obtain their form

$$
\left\{\begin{array}{l}
\frac{d x_{k}}{d \tau}=\varepsilon x_{k}\left(1-x_{k}^{2}\right)-y_{k} \\
\frac{d y_{k}}{d \tau}=x_{k} \pm \gamma(\tau) \sum_{j=1}^{2} y_{j} \quad(k=1,2 .)
\end{array}\right.
$$

In Eq. $3, \varepsilon$ denotes to the nonlinearity of van der Pol oscillator and $\gamma(\tau)$ is the characteristics of STVR.

\section{SynChronization PhenOMENA}

For the following computer simulations, we fix the circuit system parameters at $\varepsilon=2.0, \gamma=0.1$ and $\omega=1.5$. For observing the in-phase and the anti-phase synchronized solutions as shown in Figs. 6, 7, the value of the coupling strength $\gamma$ is the least critical, whereas a strong oscillator nonlinearity $\varepsilon$ is needed and $\omega$ should be chosen, under the other given parameter values, from the interval $(1.44,1.58)$. Figure 6 shows the discrete data $x_{1}-x_{2}$ between two van der Pol oscillators at $\tau=k \pi / \omega$. From this figure we can see that the switching synchronization between the in-phase and the anti-phase state is observed. The simulation result of switching phase difference is shown in Fig. 7. We obtain the phase difference when the solution of the 1st oscillator crosses the Poincaré map defined as $x_{1}<0, y_{1}=0$. It is worthwhile mentioning that for a periodically switched TVR, the switching between the two regimes can generally not be observed. For characterizing the switching by means of the sojourn time, we calculated a moving average of 30 steps of the phase difference, which was sufficient for distinguishing between the in-phase state and the anti-phase state as shown in Fig. 8. We attribute the behavior to the in-phase or to the antiphase synchronized state, respectively, if the averaged phase difference is smaller or larger than 90 degrees. The result of the statistics of the distribution among in-phase and antiphase sojourn time is shown in Fig. 9 for switching probability $p_{+}=p_{-}=0.5$. As can be expected, the difference between distributions associated with the two regimes increases if the switching probabilities are changed, see Figs. 10, 11.

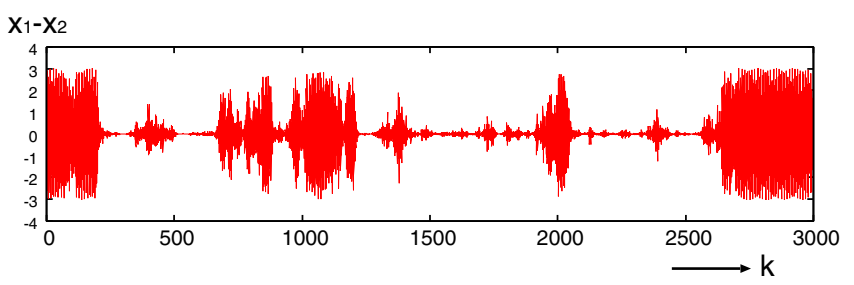

Fig. 6. Difference $x_{1}-x_{2}$ of the two coupled van der Pol oscillators with STVR with parameter $p_{+}=0.5, p_{-}=0.5$. 


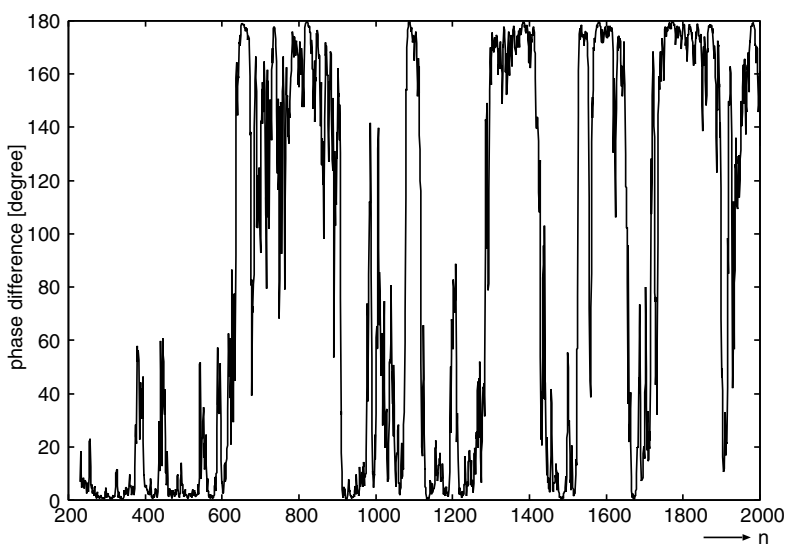

Fig. 7. Switching phase states (horizontal axis: Poincaré point $n$, vertical axis: phase difference between two van der Pol oscillators, $p_{+}=0.5, p_{-}=0.5$ ).

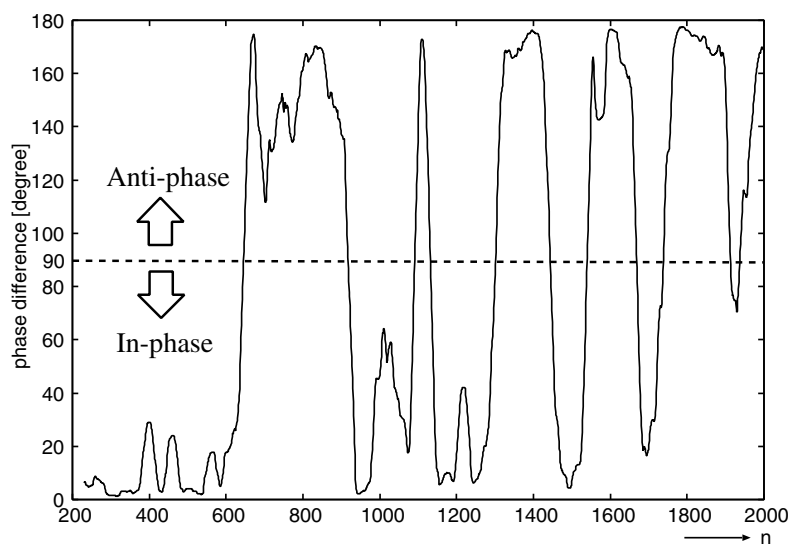

Fig. 8. Moving average of the phase states $\left(p_{+}=0.5, p_{-}=0.5\right)$.

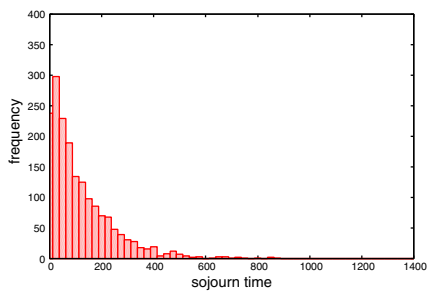

(a) In-phase.

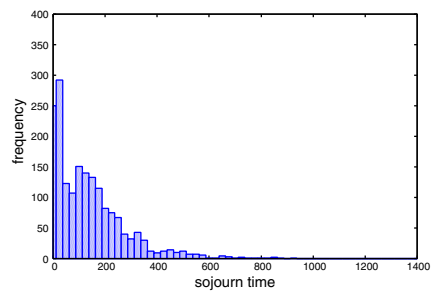

(b) Anti-phase.
Fig. 9. Frequency distribution of sojourn time $\left(p_{+}=0.5, p_{-}=0.5\right)$.

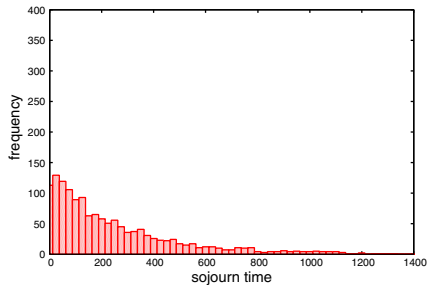

(a) In-phase.

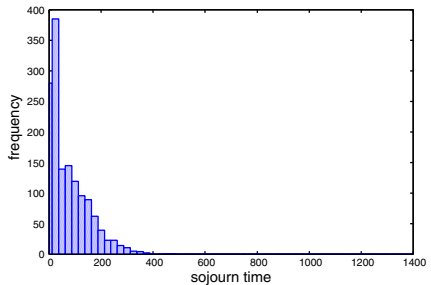

(b) Anti-phase.
Fig. 10. Frequency distribution of sojourn time $\left(p_{+}=0.48, p_{-}=0.52\right)$.

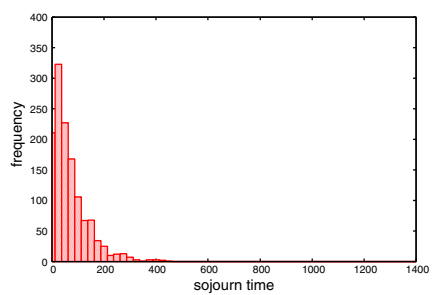

(a) In-phase.

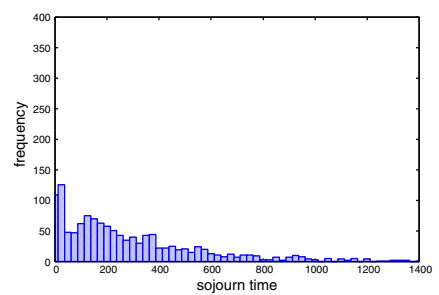

(b) Anti-phase.
Fig. 11. Frequency distribution of sojourn time for $\left(p_{+}=0.52, p_{-}=0.48\right)$.

The collected results obtained by changing the STVR probability $p_{+}$are shown in Fig. 12, where we use the averaged sojourn time to characterize the preference for one of the two regimes. When the probability of STVR $p_{+}$is 0.5 , we have about equal preference. By increasing $p_{+}$, the preference for the anti-phase regime increases and decreases for the in-phase regime. The switching phase state phenomena can be observed for $0.42 \leq p_{+} \leq 0.58$. If $p_{+}>0.58$, in-phase synchronization breaks down and only the anti-phase synchronization can be observed. For the choice $p_{+}<0.42$, in contrast, only the inphase synchronization can be observed. These findings can be collected by displaying the percentage of the in-phase state in dependence $p_{+}$. Based on simulations comprising 500,000 samples for each data point, we obtained the result as displayed in Fig. 13, showing a striking resemblance with the sigmoid function centered around $p_{+}=0.5$.

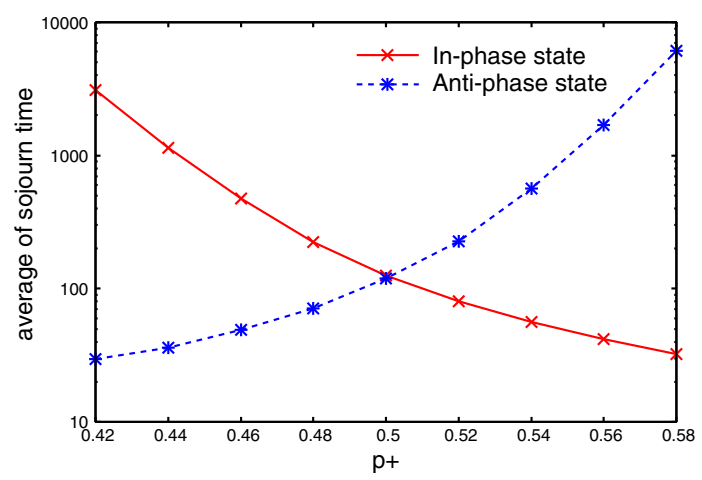

Fig. 12. Average of sojourn time in dependence on $p_{+}$.

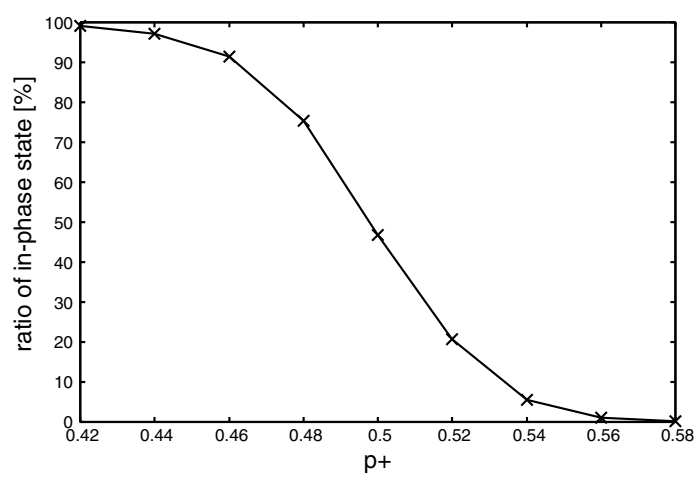

Fig. 13. Ratio of in-phase synchronization state. 


\section{INFLUENCE OF NOISE}

In realistic systems, noise is omnipresent. Therefore, it is important to study the effect of noise on our findings. When extended by noise contributions to the voltage of the two capacitances, the normalized circuit equations with noise are described by

$$
\left\{\begin{array}{l}
\frac{d x_{k}}{d \tau}=\varepsilon x_{k}\left(1-x_{k}^{2}\right)-y_{k}+D \xi_{k} \\
\frac{d y_{k}}{d \tau}=x_{k} \pm \gamma(\tau) \sum_{j=1}^{2} y_{j} \quad(k=1,2)
\end{array}\right.
$$

where $\xi_{k}$ denotes the Gaussian white noise and $D$ is the noise intensity. The two complementary behaviors are now investigated at the switching probability $p_{+}=0.5$, where we are also interested in the influence that a correlation between the two noise contributions would have. As a function of the percentage of shared vs. independently chosen noise contributions, we obtain the results as displayed in Figs.14, 15.

For independent noise contributions, we observe a stochastic resonance phenomenon for in-phase and anti-phase synchronization regimes. Namely, the length of the average sojourn time becomes longer by adding weak noise. This happens at considerable, but not dominant, noise strengths, in the case that the noise contributions at the capacitances are independent. As a function of the percentage of noise contributions randomly chosen vs. shared among the capacitances, the stochastic resonance phenomenon increases in the in-phase regime. The dependence clearly indicates that the sharing acts as if an additional connection among the two oscillators were established. For fully correlated (shared) noise sources, upon increasing the strength of the noise, this 'connection' first supports and then overwhelms the stochastic resonance phenomenon. In the anti-phase synchronization regime, the effect is contrary: the connection naturally works against the stochastic resonance phenomenon.

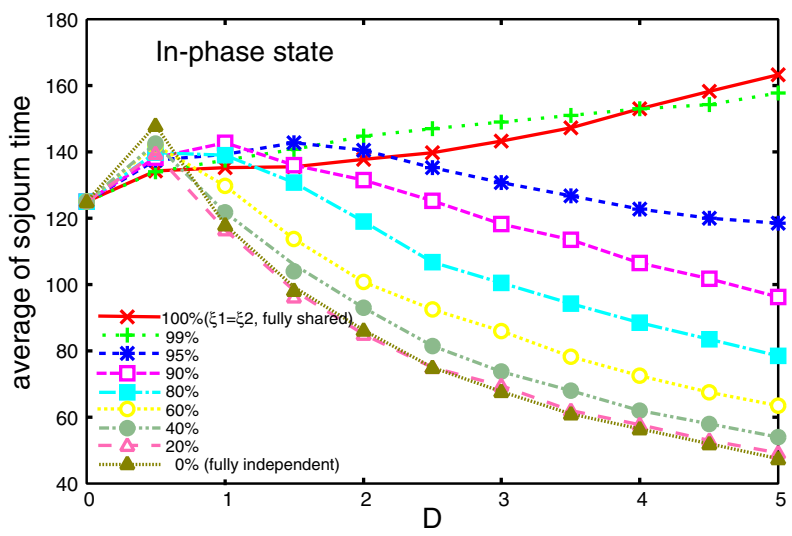

Fig. 14. Average sojourn time of in-phase by changing noise intensity.

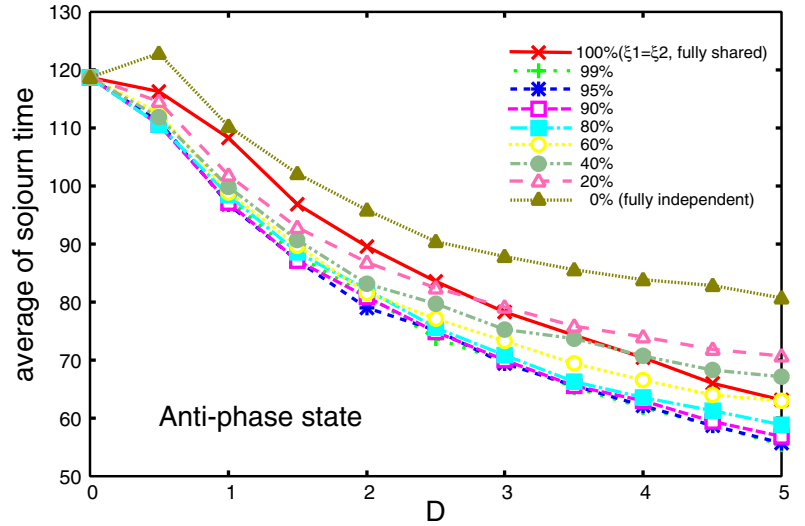

Fig. 15. Average sojourn time of anti-phase by changing noise intensity.

\section{CONCLUSION}

In this study, we have investigated the switching phase state of two van der Pol oscillators coupled by STVR. We have analyzed the statistics of the switching, with and without additional noise. The stochastic resonant behavior of synchronization was observed when the strength of the adding noise is small. We have confirmed that correlating the two noise sources quickly destroys the stochastic resonance phenomenon in the anti-phase synchronization regime.

\section{REFERENCES}

[1] L.L. Bonilla, C.J. Perez Vicente and R. Spigler, "Time-periodic phases in populations of nonlinearly coupled oscillators with bimodal frequency distributions," Physica D: Nonlinear Phenomena, vol.113, no.1, pp.7997, Feb. 1998.

[2] J.A. Sherratt, "Invading wave fronts and their oscillatory wakes are linked by a modulated traveling phase resetting wave," Physica D: Nonlinear Phenomena, vol.117, no.1-4, pp.145-166, June 1998.

[3] G. Abramson, V.M. Kenkre and A.R. Bishop, "Analytic solutions for nonlinear waves in coupled reacting systems," Physica A: Statistical Mechanics and its Applications, vol.305, no.3-4, pp.427-436, Mar. 2002.

[4] I. Belykh, M. Hasler, M. Lauret and H. Nijmeijer, "Synchronization and graph topology," Int. J. Bifurcation and Chaos, vol.15, no.11, pp.34233433, Nov. 2005.

[5] C.M. Gray, "Synchronous oscillations in neural systems: mechanisms and functions," J. Computational Neuroscience, vol.1, pp.11-38, 1994.

[6] R. Stoop and C. Wagner, "Neocortex's architecture optimizes computation, information transfer and synchronizability, at given total connection length," International Journal of Bifurcation and Chaos, vol.17, no.7, pp.2257-2279, 2007.

[7] T. Suezaki and S. Mori, "Mutual synchronization of two oscillators," Trans. IECE, vol.48, no.9, pp.1551-1557, Sep. 1965.

[8] H.B. Fotsina and J. Daafouza, "Adaptive synchronization of uncertain chaotic colpitts oscillators based on parameter identification" Physics Letters A, vol.339, pp.304-315, May. 2005.

[9] I. Belykh, V. Belykh and M. Hasler, "Blinking model and synchronization in small-world networks with a time-varying coupling," Physica D, vol.195, no.1-2, pp.188-206, 2004.

[10] Y. Nishio and S. Mori, "Chaotic phenomena in nonlinear circuits with time-varying resistors," IEICE Trans. Fundamentals, vol.E76-A, no.3, pp.467-475, Mar. 1993.

[11] C. Huygens, J. Scavants XI, 79 (1665); XII, 86 (1665). For a recent review of synchronization processes see S. Strogatz, Sync: The Emerging Science of Spontaneous Order (Hyperion, New York, 2003).

[12] Y. Uwate and Y. Nishio, "Synchronization phenomena in van der Pol oscillators coupled by a time-varying resistor," International Journal of Bifurcation and Chaos, vol.17, no.10, pp.3565-3569, 2007.

[13] Y. Uwate and Y. Nishio, "Switching phase states of chaotic circuits coupled by time-varying resistor," Proceedings of ISCAS'07, pp. 17831786, May 2007. 